\title{
Etiología del acúfeno pulsátil. Caso clínico
}

\section{Etiology of pulsatile tinnitus. Case report}

\author{
Lorena Galindo I. ${ }^{1,2}$
}

\begin{abstract}
Resumen
El acúfeno es un síntoma relativamente frecuente en una consulta de otorrinolaringología. Se han descrito interacciones en las células ciliadas externas o internas, desequilibrios en el balance de las fibras aferentes y fenómenos de reorganización cortical tras lesiones periféricas que están involucrados en un 90\%-95\% de las causas del acúfeno. El restante 5\%-10\% está constituido por un tipo de acúfenos llamados objetivos, que no comparten estos mecanismos fisiopatológicos, sino que se originan en alguna estructura del organismo generalmente ajena a la vía auditiva y estimulan el aparato auditivo igual que lo haría un sonido del exterior. Presentamos el caso de un varón de 52 años remitido al Servicio de Otorrinolaringología de nuestro hospital por acúfeno pulsátil de meses de evolución, sin asociar hipoacusia, ni vértigo, ni otra sintomatología.

Palabras clave: acúfeno pulsátil, otorrinolaringología.
\end{abstract}

\begin{abstract}
Tinnitus is a relatively frequent symptom in an otolaryngology consultation. Interactions in external or internal hair cells, imbalances in the afferent fiber balance and cortical reorganization phenomena after peripheral injuries have been described in 90\%-95\% of the causes of tinnitus. The remaining 5\%-10\% is comprised of a type of tinnitus called objective, which do not share these pathophysiological mechanisms, but originate from some structure of the body generally external to the auditory pathway and stimulate the auditory apparatus just as a sound from the exterior. We present the case of a 52-year-old man referred to the Otolaryngology service at our hospital for pulsatile tinnitus of months of evolution, with no hearing loss, vertigo, or other symptoms associated.

Keywords: pulsatile tinnitus, otolaryngology.
\end{abstract}

${ }^{1}$ Hospital Universitario Morales Meseguer, Murcia, España. ${ }^{2}$ Hospital General Universitario Reina Sofía, Murcia, España.

La autora declara no tener conflictos de interés.

Recibido el 13 de abril de 2020. Aceptado el 22 de abril de 2020.

Correspondencia: Lorena Galindo I.

Servicio de

Otorrinolaringología.

Hospital Universitario Morales Meseguer. Hospital Universitario Reina Sofía.

Murcia, España.

Email: lorenagi91@hotmail. com

\section{Introducción}

El acúfeno es un motivo de consulta relativamente frecuente para el especialista en Otorrinolaringología. La mayoría de ocasiones se trata de un síntoma no objetivable ${ }^{1}$, sin embargo, hasta el 5-10\% de los acúfenos se presenta con características pulsátiles, pudiendo enmascarar otras patologías ${ }^{2}$. El diagnóstico etiológico será fundamental a la hora de poder descartar posibles patologías y sus implicaciones diagnósticas. Según la presencia o ausencia de percepción del sonido por parte del examinador, clasificaremos los acúfenos en objetivos y subjetivos ${ }^{3,4}$. Así, el porcentaje de acúfenos pulsátiles registrado tiene, salvo excepciones, origen vascular, teniendo como origen las vibraciones del flujo sanguíneo resultado de estructuras vasculares intracraneanas que estimulan el aparato auditivo con una frecuencia similar al pulso arterial ${ }^{3}$.

\section{Epidemiología y fisiopatogenia}

El acúfeno pulsátil es más frecuente en el género femenino y la mayoría son unilaterales, sin embargo, hasta $15 \%$ de los pacientes refieren tinnitus pulsátil de forma bilateral ${ }^{4}$. Existen multitud de patologías descritas que pueden causar acúfeno pulsátil. Hoffman y cols. ${ }^{1}$, realizaron una revisión de 563 pacientes en 6 series de casos publicados y 77 pacientes estudiados en su centro, clasificando la etiología del acúfeno pulsátil en cuanto a localización y causa (Tabla 1). 
Tabla 1. Clasificación etiológica del acúfeno pulsátil según localización'

\begin{tabular}{cccc}
\hline Arterial & Arteriovenosa & Venosa & Otras \\
$\mathbf{2 1 , 7 4 \%}$ & $\mathbf{2 1 , 1 2 \%}$ & $\mathbf{2 6 , 6 3 \%}$ & $\mathbf{3 0 , 1 8 \%}$ \\
Estenosis arterial & Fístula arteriovenosa & $\begin{array}{c}\text { Hipertensión intracraneal } \\
\text { benigna }\end{array}$ & $\begin{array}{c}\text { Dehiscencia del canal } \\
\text { semicircular }\end{array}$ \\
Malformaciones & $\begin{array}{c}\text { Malformaciones (carótida } \\
\text { interna aberrante) }\end{array}$ & $\begin{array}{c}\text { Malformaciones } \\
\text { (dehiscencia del bulbo de la } \\
\end{array}$ & yugular) \\
\hline
\end{tabular}

En una revisión retrospectiva de 84 pacientes con acúfeno pulsátil se encontró que el $42 \%$ tenía asociado una patología vascular (lo más frecuente era la fístula arteriovenosa $)^{5}$. La mayoría de estos pacientes no tienen otros síntomas otológicos (hipoacusia, vértigo, plenitud auditiva) $y$, al igual que con otras causas, escuchan más el acúfeno en ambientes poco ruidosos. Las fístulas arteriovenosas asocian generalmente un acúfeno ipsilateral a la fístula ${ }^{6}$. Éstas se asocian frecuentemente con trombosis que puede estar asociada a otros procesos como infecciones, tumores, traumatismos craneoencefálicos o intervenciones previas. El diagnóstico y el tratamiento precoz a la hora de descartar una fístula arteriovenosa son primordiales, debido al riesgo de complicaciones que conlleva. Otra etiología de interés es el conflicto neurovascular. La anatomía del complejo neurovascular a nivel del ángulo pontocerebeloso es extremadamente variable, lo que puede afectar al desarrollo de la arteria cerebelosa anteroinferior (AICA) y la arteria cerebelosa posteroinferior (PICA). Esta situación puede repercutir en la compresión de los nervios craneales a nivel de la fosa cerebral posterior. De esta forma, la compresión vascular del nervio vestíbulococlear en el ángulo pontocerebeloso a consecuencia de trayectos aberrantes de la AICA, PICA o alguna de sus ramas puede manifestarse con una sintomatología variada, incluyendo hipoacusia, vértigo y acúfenos?.

Otra patología vascular que puede ocasionar acúfeno pulsátil son los paragangliomas timpánicos, anteriormente conocidos como tumores del glomus timpánico, y los paragangliomas del bulbo yugular, comúnmente conocidos como glomus yugular. Se trata de neoplasias benignas vasculares que se encuentran alrededor de la bifurcación carotídea, dentro del bulbo yugular o a lo largo de las arterias timpánicas en el oído medio. Estos tumores causan frecuentemente acúfeno pulsátil unilateral y, en gran parte de los casos, asocian en la exploración una imagen de masa rojiza o azul visible tras la membrana timpánica. Además, a medida que aumentan de tamaño, pueden causar hipoacusia y alternación de otros pares craneales. Se describen otras causas de acúfeno pulsátil como situaciones de aumento de la presión intracraneal y dehiscencia del bulbo yugular ${ }^{6}$, que puede disminuir o detenerse aplicando presión sobre la vena yugular o con los movimientos cefálicos.

El acúfeno pulsátil también puede tener origen muscular, a consecuencia del espasmo de uno o ambos músculos del oído medio (el tensor del tímpano y el músculo estapedio), inervados por los pares craneales V y VII, respectivamente. Estos espasmos pueden ocurrir debido a una enfermedad otológica, pero también se han descrito en enfermedades neurológicas como la esclerosis múltiple. Así mismo, el mioclono de la musculatura palatina que se une al orificio de la trompa de Eustaquio también puede producir chasquidos o pulsaciones irregulares o rápidas, pudiendo ser percibidas por el paciente como acúfeno pulsátil. Esta causa se ha descrito asociada a otras patologías como la esclerosis múltiple y la neuropatía relacionada con las causas tóxicas o metabólicas ${ }^{6}$.

\section{Diagnóstico}

Para el diagnóstico de pacientes con acúfeno pulsátil es fundamental realizar una historia clínica detallada. La anamnesis debe 
incluir una descripción del acúfeno (pulsátil o no pulsátil, ritmo, tono, cambios con movimientos cefálicos o compresión cervical) y si existen factores desencadenantes. Es esencial describir si el paciente padece otras enfermedades o cirugías previas. Se debe documentar si existen antecedentes personales de patología otológica, traumatismos craneoencefálicos y uso de fármacos y tóxicos ${ }^{8}$. Se debe realizar una exploración física completa, incluyendo la exploración de pares craneales, otoscopía y pruebas audiométricas en todos los pacientes. Si se sospecha origen vascular, debe realizarse auscultación cervical, del área periauricular y de la mastoides en diferentes posiciones, teniendo especial atención ante cualquier cambio con la compresión cervical. Debido a que el acúfeno pulsátil puede estar relacionado con otras patologías de mayor relevancia clínica, los pacientes con alta sospecha deben ser evaluados exhaustivamente con el objetivo de descartar una lesión del sistema nervioso central, como una fístula arteriovenosa dural, malformación arteriovenosa o aneurisma, o un tumor en la base del cráneo ${ }^{8}$. El gold estándar para el diagnóstico de la fístula arteriovenosa es la angiografía. Estas lesiones a menudo también se pueden diagnosticar de forma no invasiva con angiorresonancia magnética o angiografía por tomografía computarizada ${ }^{9}$. La resonancia magnética puede diagnosticar patología de origen central asociada a la presencia de acúfeno pulsátil ${ }^{10}$.

\section{Caso Clínico}

Presentamos el caso de un varón de 52 años remitido al Servicio de Otorrinolaringología de nuestro hospital por acúfeno pulsátil de oído derecho de meses de evolución, sin asociar hipoacusia, ni vértigo, ni otra sintomatología. A la exploración física, el oído derecho presentaba integridad de membrana timpánica sin objetivar aparente contenido en caja ni otros hallazgos de interés. La audiometría estaba dentro de la normalidad. Se realizó una ecografía de troncos supraaórticos sin evidenciar alteraciones de interés. Se solicitó una resonancia magnética que informó de un bucle de la arteria cerebelosa anteroinferior (AICA) intracanalicular (Figura 1). Debido a la ausencia de otra sintomatología y al riesgo asociado al tratamiento se optó por manejo conservador con psicoeducación.

\section{Discusión}

Ante cualquier paciente con acúfeno pulsátil debe realizarse un diagnóstico exhaustivo con el objetivo de descartar patologías asociadas de importante relevancia clínica. La evaluación de estos pacientes requiere de una anamnesis y exploración física detallada, que en muchos casos requerirá de otras pruebas diagnósticas como la angiorresonancia magnética ${ }^{11}$. La etiología más frecuente del acúfeno pulsátil es la vascular. El bucle de la arteria cerebelosa anteroinferior (AICA) es una malformación vascular que, si bien se presenta desde el nacimiento, puede ser asintomática y únicamente dar clínica con el paso del tiempo. El nervio facial (VII) y el vestibulococlear (VIII) emergen del ángulo pontocerebeloso y en su recorrido ingresan en el conducto auditivo interno. Una de las estructuras vasculares que mantienen relación con estos es la AICA.

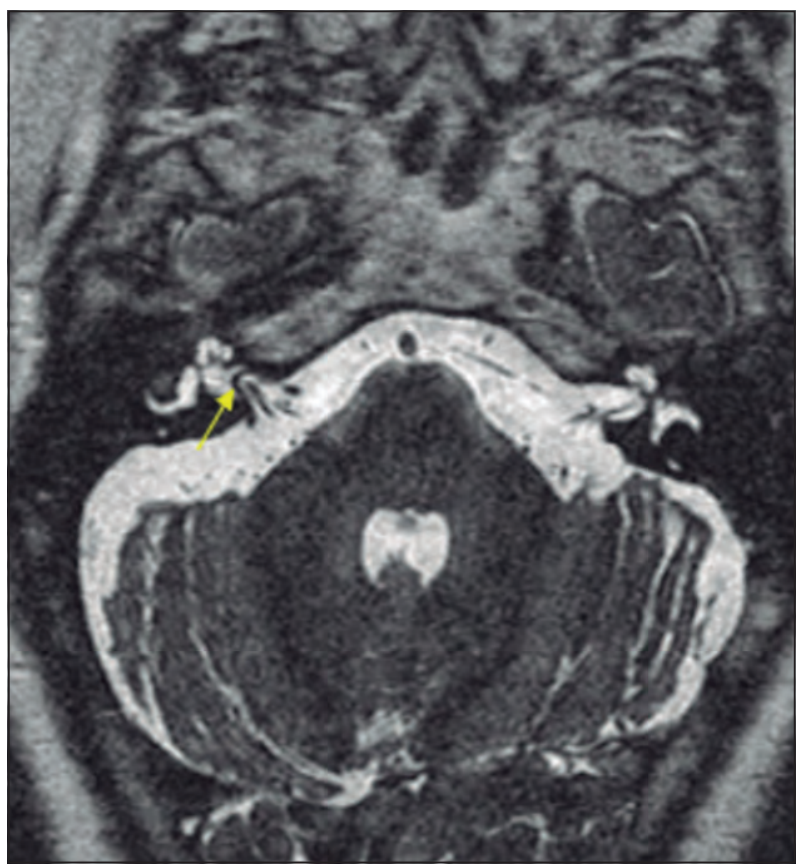

Figura 1. Bucle de la arteria cerebelosa anteroinferior (AICA) intracanalicular. 
Tabla 2. Clasificación de las asas vasculares ${ }^{14}$

\begin{tabular}{ll}
\hline Clasificación anatómica & Clasificación según contacto \\
Tipo I & Categoría A \\
Sin contacto con el CAl & No hay contacto \\
Tipo II & Categoría B \\
Entra en el CAl, pero no se extiende $>50 \%$ de la longitud & $\begin{array}{l}\text { Asa vascular que se extiende directamente adyacente al } \\
\text { nervio }\end{array}$ \\
Tipo III & Categoría C \\
Se extiende $>50 \%$ de la longitud del CAI & Bucle entre el VII y el VIII pares craneales \\
& Categoría D \\
& Asa que desplaza el nervio \\
\hline
\end{tabular}

En el conducto auditivo interno hay un espacio mínimo para el nervio facial, vestibulococlear y la arteria cerebelosa anteroinferior, generando una susceptibilidad anatómica de compresión vascular y alteración de dichos pares cranea$l s^{12}$. El principal síntoma percibido por los pacientes con bucles de la AICA es el acúfeno (hasta en un 65\% de los casos, en comparación con el $10 \%$ de la población general) y su forma de presentación es en la mayoría de casos pulsátil $^{13}$. Ante la sospecha, es necesario solicitar estudios de imagen como la resonancia magnética. En ésta, es posible identificar los bucles de la AICA. Gorrie A. y cols. ${ }^{14}$ presentaron una clasificación de las asas vasculares (Tabla 2).

Según la misma, en aproximadamente el 9\% de los casos, las asas vasculares de la AICA se extienden más del $50 \%$ de la longitud del conducto auditivo interno y $12,3 \%$ se producen dentro del conducto auditivo interno ${ }^{13}$. A pesar de estos hallazgos, la afirmación de que la causa de los síntomas otológicos es la compresión vascular es aún es controvertida. Algunos estudios defienden que el contacto entre el VIII par craneal y el asa vascular puede considerarse un hallazgo dentro de la normalidad ${ }^{15}$ y serían necesarios más estudios al respecto. Respecto al tratamiento, se han recogido buenos resultados en la disminución del acúfeno con la cirugía de descompresión microvascular del VIII par craneal en el ángulo pontocerebeloso, si bien, tal y como se ha mencionado en la actualidad el contacto entre el asa vascular y el VIII par craneal puede llegar a considerarse como un hallazgo anatómico normal ${ }^{15}$. Si bien existen actualmente otras pruebas complementarias que aportan información precisa ante pacientes con sospecha de etiología vascular, tales como la angiografía por tomografía computarizada y la angiorresonancia magnética, en la actualidad la resonancia magnética cuenta con múltiples ventajas al no ser invasiva y no someter al paciente a radiación. En el caso que presentamos se optó por estudiar al paciente con resonancia magnética debido a sus comorbilidades y la mayor disponibilidad en nuestro centro.

\section{Conclusión}

El acúfeno es un síntoma otológico muy frecuente, en que entre el 5\% a 10\% tiene características pulsátiles, pudiendo enmascarar otras patologías. Es por esto que un diagnóstico etiológico es fundamental por las posibles implicaciones que esta entidad puede conllevar. En la mayoría de las ocasiones el origen es vascular y requiere una evaluación exhaustiva. El diagnóstico diferencial incluye las malformaciones arteriovenosas, las fístulas arteriovenosas, los soplos y tumores como el paraganglioma. Por todo ello, ante todo paciente con acúfeno de características complejas se debe realizar una historia clínica detallada, una exploración que incluya exploración de pares craneales, otoscopía y pruebas audiométricas. Así mismo, se deben solicitar pruebas de imagen como resonancia magnética, tomografía computarizada y/o angiografía ante cualquier paciente que suscite dudas diagnósticas. 


\section{Bibliografía}

1. Hofmann E, Behr R, Neumann-Haefelin T, Schwager K. Pulsatile Tinnitus: Imaging and Differential Diagnosis. Dtsch Arztebl Int 2013;110:451-458.

2. Mattox D, Hudgins P. Algorithm for evaluation of pulsatile tinnitus. Acta Otolaryngol 2008;128:427-431.

3. Herraiza C, Aparicioa JM. Claves diagnósticas en los somatosonidos o acúfenos pulsátiles. Acta Otorrinolaringol Esp 2007;58:426-433.

4. Shin E, Lalwani A, Dowd C. Role of Angiography in the Evaluation of Patients With Pulsatile Tinnitus. Laryngoscope 2000;110:1916-1920.

5. Waldvogel D, Mattle HP, Sturzenegger M, Schroth G. Pulsatile tinnitus-a review of 84 patients. J Neurol 1998;245:137-142.

6. Fortune DS, Haynes DS, Hall JW 3rd. Tinnitus. Current evaluation and management. Med Clin North Am 1999;83:153-162.

7. Wuertenberger CJ, Rosahl SF. Vertigo and Tinnitus Caused by Vascular Com-pression of the Vestibulocochlear Nerve, Not Intracanalicular Vestibular Schwannoma: Review and Case Presentation. Skull Base 2009;19:417-424.

8. Herraiz C. Mecanismos fisiopatológicos en la génesis y cronificación del acúfeno. Acta Otorrinolaringol Esp 2005;56:335-342.
9. Mundada P, Singh A, Lingam RK. CT arteriography and venography in the evaluation of Pulsatile tinnitus with normal otoscopic examination. Laryngoscope 2015;125:979-984.

10. Forsting M. CTA of the ICA bifurcation and intracranial vessels. Eur Radiol 2005;15:D25-D27.

11. Shweel M, Hamdy B. Diagnostic utility of magnetic resonance imaging and magnetic resonance angiography in the radiological evaluation of pulsatile tinnitus. Am J Otolaryngol 2013;34:710-717.

12. Shulman A. Clinical types of tinnitus. En: Shulman A, editor. Tinnitus diagnosis/treatment. San Diego: Singular; 1997. p. 329-41.

13. Roldán Fridalgo A, Rodríguez Valiente A, Martín Gonzalez F, Pinilla M, Trinidad A, Brea Álvarez B. Vascular loops and neuro-otologic symptons. Is there any correlation? Int J Otorhinolaryngol Head Neck Surg 2013;2:145-7.

14. Gultekin S, Celik H, Akpek S, Oner Y, Gumus T, Tokgoz N. Loops at the Cerebellopontine Angle: Is there a Correlation with Tinnitus? Am J Neuroradiol 2008;29:1746-49.

15. Borghei Razavi H, Darvish O, Schick U. Disabling vértigo and tinnitus caused by intrameatal compression of the anterior cerebellar artery on the vestibulocochlear nerve: a case report. J Neurol Surg Rep 2014;75:e47-e51. 